\title{
Le manchon souple d'étranglement, organe autorégulateur de répartition des débits constants dans les canaux d'irrigation
}

\author{
The throttle hose, an automatic diversion device \\ with constant discharge for irrigation channels
}

\author{
D. Vischer \\ Laboratoire de recherches hydrauliques, hydrologiques et glaciologiques \\ de l'École polytechnique fédérale de Zurich \\ P. Peter \\ Universal Ingénieurs Conseils SA, Bâle
}

Avec le manchon souple d'étranglement, on dispose d'un nouvel organe autorégulateur de répartition des débits constants dans les réseaux d'irrigation. Sa configuration est simple, ce qui se traduit par des coûts modestes de réalisation.

Le présent article explique le principe de son fonctionnement et décrit les essais de laboratoire menés avec une série de prototypes. Il traite également certains problèmes spécifiques de ce type d'appareillage. Enfin, il propose un procédé de dimensionnement basé sur un diagramme simple.

The throttle hose is a new automatic diversion device with constant discharge for irrigation networks. Its configuration is simple, resulting in moderate implementation costs. This paper describes the theory of operation and discusses laboratory tests conducted on a series of prototypes. It also covers a few problems specific to this type of device. Finally, it proposes a dimensioning process based on a simple diagram.

\section{Principe du manchon souple d'étranglement}

Le principe est présenté dans la figure $l$ (voir Vischer 1979) : Un bassin est pourvu d'un orifice équipé d'un tuyau qui se prolonge à l'intérieur de la retenue, où ce dernier est soumis à une surpression extérieure. Sous cette sollicitation, le tuyau se voile et sa section subit un étranglement. De ce fait, le débit d'écoulement diminue. $\mathrm{Si}$ la déformation engendrée est élastique, l'étranglement varie avec la surpression et par conséquent avec le niveau d'eau dans le bassin. L'étranglement est d'autant plus accentué que ce niveau d'eau est élevé.

L'effet d'étranglement recherché peut être favorisé par le choix d'un matériau approprié pour le tuyau. L'idée s'impose naturellement de remplacer une partie du tuyau par un manchon souple qui se voile sous une faible charge et provoque ainsi l'étranglement. Cet effet est particulièrement marqué quand la section d'étranglement coïncide avec la section de sortie du jet, c'est-à-dire lorsque le jet décolle de la paroi du manchon immédiatement après la section minimale.

Lors du remplissage du bassin, le manchon présente successivement différents comportements. D'abord, il n'est que partiellement rempli et assure un écoulement à surface libre. Ensuite, le manchon se remplit complètement et commence à fonctionner en charge. Il est susceptible de résister jusqu'à une certaine valeur de la surpression extérieure et sa forme reste cylindrique. L'écoulement se

LA HOUILLE BLANCHE/N²-1985 


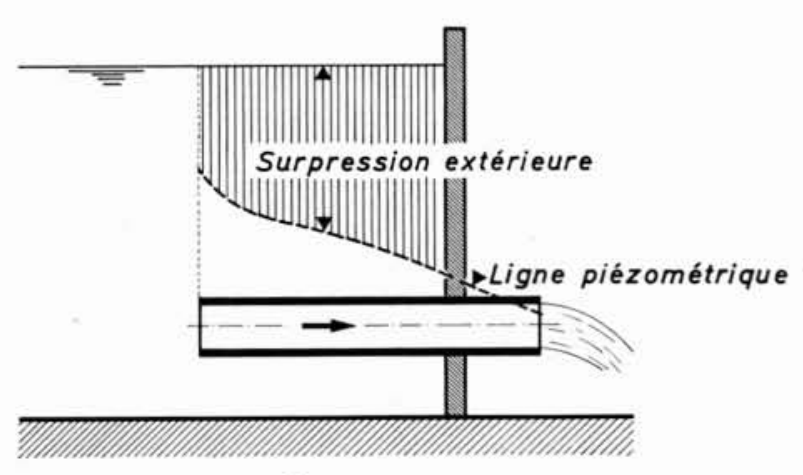

Figure 1. - Écoulement d'un bassin par un orifice équipé d'un tuyau. Schéma de la ligne piézométrique et de la surpression extérieure.

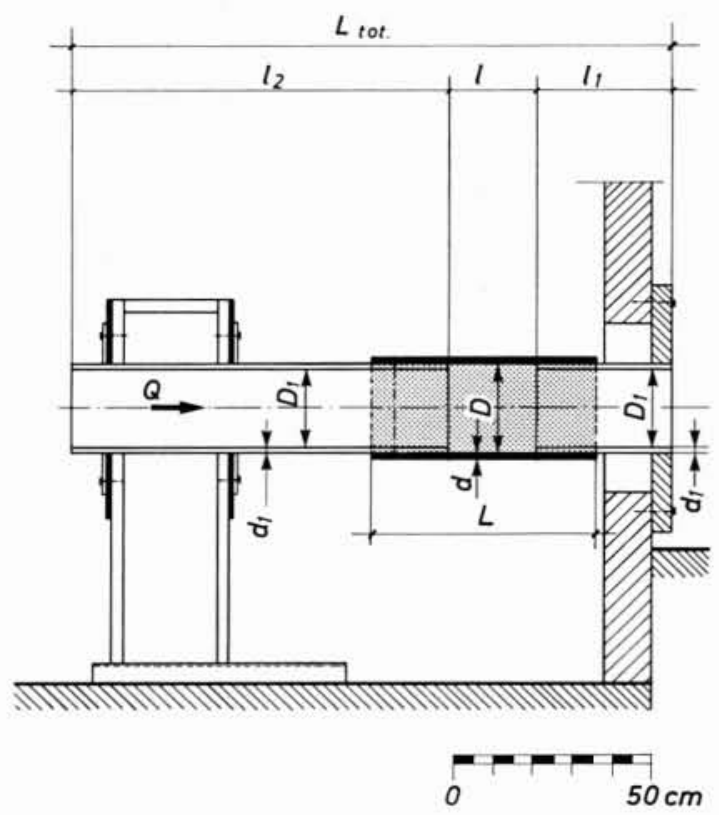

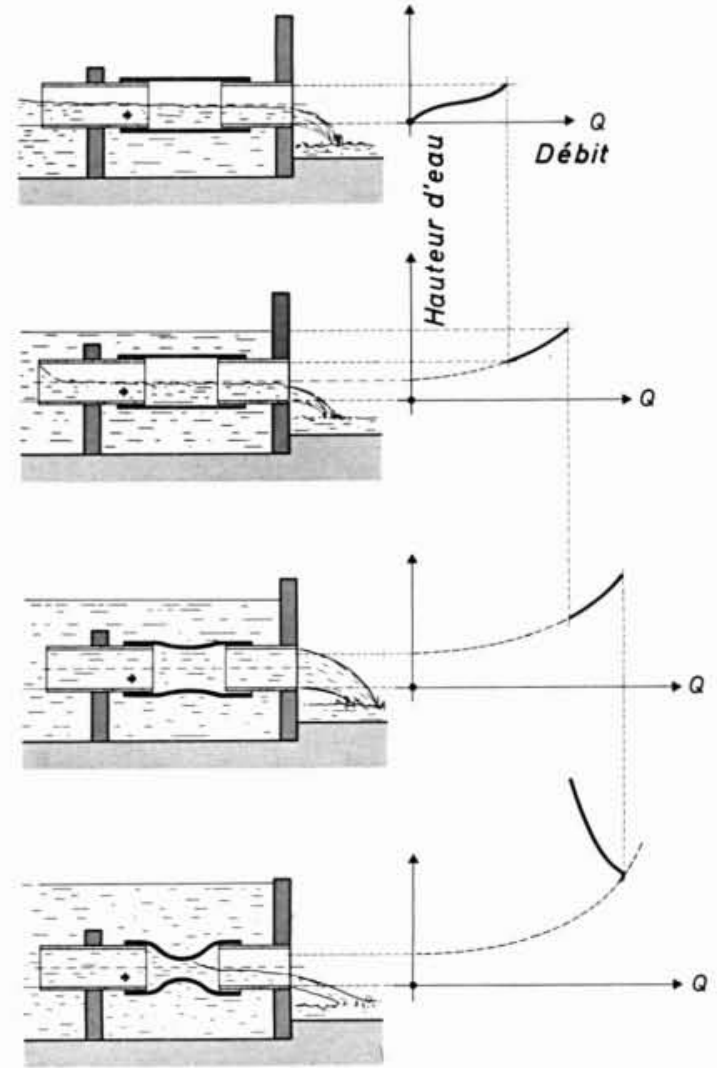

Figure 2. - Les quatre modes de fonctionnement du manchon souple d'étranglement et les courbes des débits correspondantes (représentation schématique).

Figure 3. - Coupe longitudinale du canal de $1 \mathrm{~m}$ de largeur utilisé pour la réalisation des essais. A gauche, on remarque le support ajustable qui permet d'adapter différentes longueurs des manchons. A droite, le support est fixé dans la paroi de séparation entre le niveau amont et aval. produit alors comme dans un simple tuyau. Au-delà de cette valeur critique de surpression, le manchon se voile et l'effet d'étranglement se manifeste. Fondamentalement, la courbe de débit d'un tel manchon se compose de quatre parties (figure 2):

- écoulement à surface libre;

- écoulement à surface libre avec entrée noyée;

- écoulement en charge dans le manchon non déformé;

- écoulement en charge dans le manchon déformé.

Les applications pratiques sont basées sur les caractéristiques de l'écoulement en charge dans le manchon déformé. Le choix d'une configuration appropriée du manchon souple permet d'assurer un débit pratiquement constant, indépendamment du niveau d'eau dans le bassin. Ce dispositif peut être utilisé à des fins diverses : pour la régulation de petits bassins de rétention de crues, respectivement de précipitations ou encore dans des installations d'irrigation.
Seule l'application comme organe de répartition des débits dans les réseaux d'irrigation de surface est traitée dans le cadre du présent article. Il s'agit dans ce cas de prélever un débit constant et réglable à partir d'un canal ou d'un bassin dans lequel le niveau varie, tout en maintenant les pertes de charge aussi faibles que possible.

Ce problème peut être résolu en équipant les ouvrages de répartition d'un ou de plusieurs manchons souples d'étranglement. Il en résulte un dispositif différent de celui de la figure 1 dans lequel:

- le bassin est remplacé par le canal d'adduction;

- le manchon souple d'étranglement est habituellement noyé dans le canal d'irrigation proprement dit;

- la différence de niveau d'eau entre le canal d'adduction (par exemple : canal secondaire) et le canal d'irrigation (canal tertiaire), c'est-à-dire entre les niveaux amont et aval du manchon, peut être faible. 


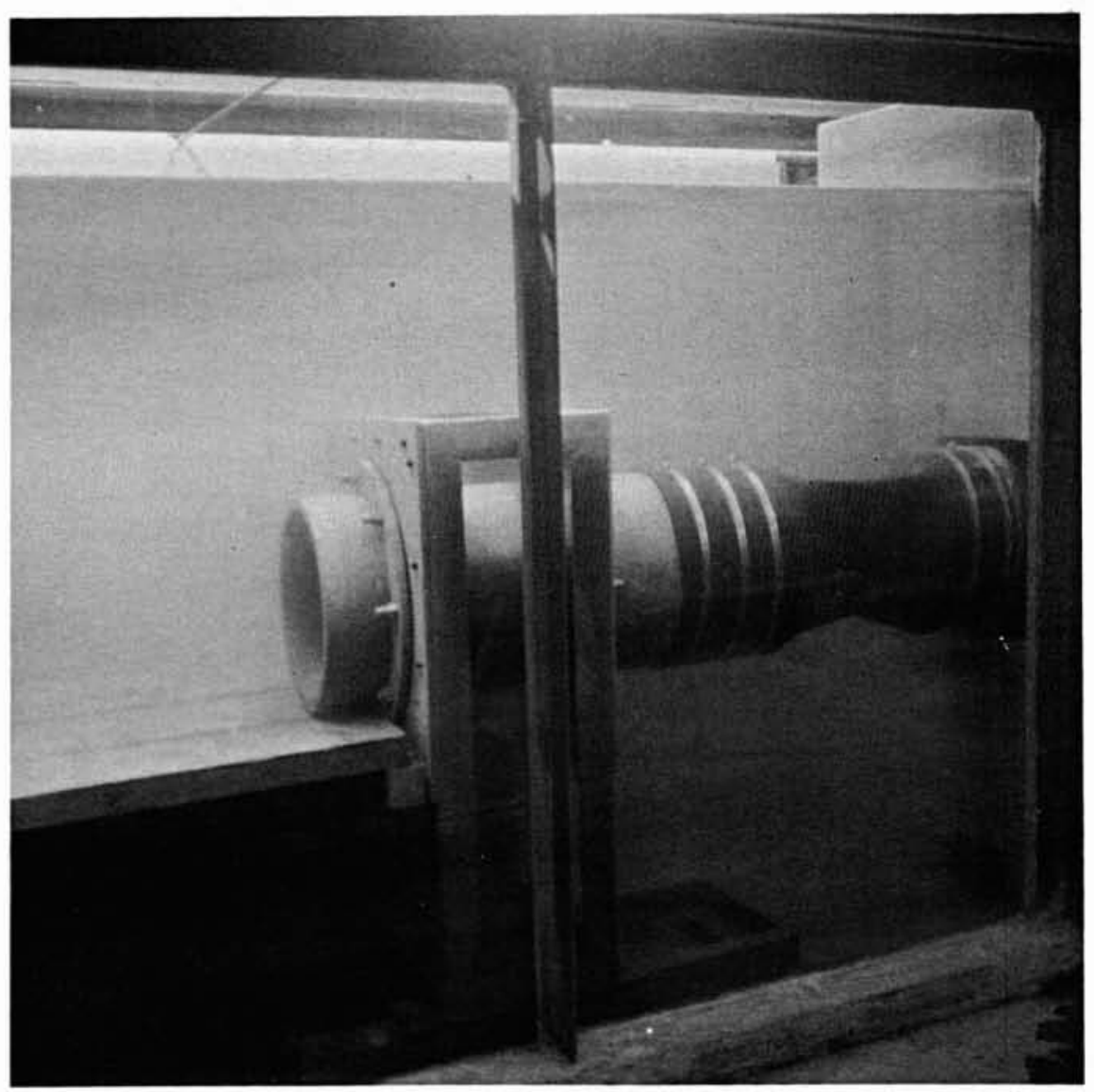

Figure 4. - Vue d'ensemble du dispositif, avec manchon souple d'étranglement du type VI en opération.

\section{Recherches en laboratoire}

En novembre 1979, la société Universal Ingénieurs-Conseils SA à Bâle, a chargé le Laboratoire de recherches hydrauliques, hydrologiques et glaciologiques de l'École polytechnique fédérale, Zurich, de vérifier les modalités d'application du manchon souple d'étranglement dans le cadre des réseaux d'irrigation. A cet effet, un ouvrage de répartition a été conçu pour des débits de 10 à $80 \mathrm{l} / \mathrm{s}$ avec une tolérance de $\pm 10 \%$, et une différence maximale entre les niveaux amont et aval de $0.70 \mathrm{~m}$.

Les essais ont été réalisés à l'échelle $1: 1$ avec un dispositif relativement simple (voir figures 3 et 4 ). La pièce principale du dispositif d'étranglement comprend deux tuyaux horizontaux en fibrociment reliés par un cylindre en caoutchouc. Ce dernier, le manchon souple d'étranglement proprement dit, a été enfilé sans précontrainte sur les tuyaux et maintenu à l'aide de brides. Le matériau du manchon consiste en un caoutchouc au Nitril d'une dureté de $49^{\circ}$ selon Shore et d'un allongement à la rupture de $450 \%$. Il se présente à l'achat sous forme de panneaux de 1 à $6 \mathrm{~mm}$ d'épaisseur qui ont été ensuite découpés et enroulés. Les deux bords assemblés, qui se recouvrent sur $20 \mathrm{~mm}$, ont été soudés à froid. Le diamètre intérieur du manchon correspond exactement au diamètre extérieur des tuyaux en fibrociment. 


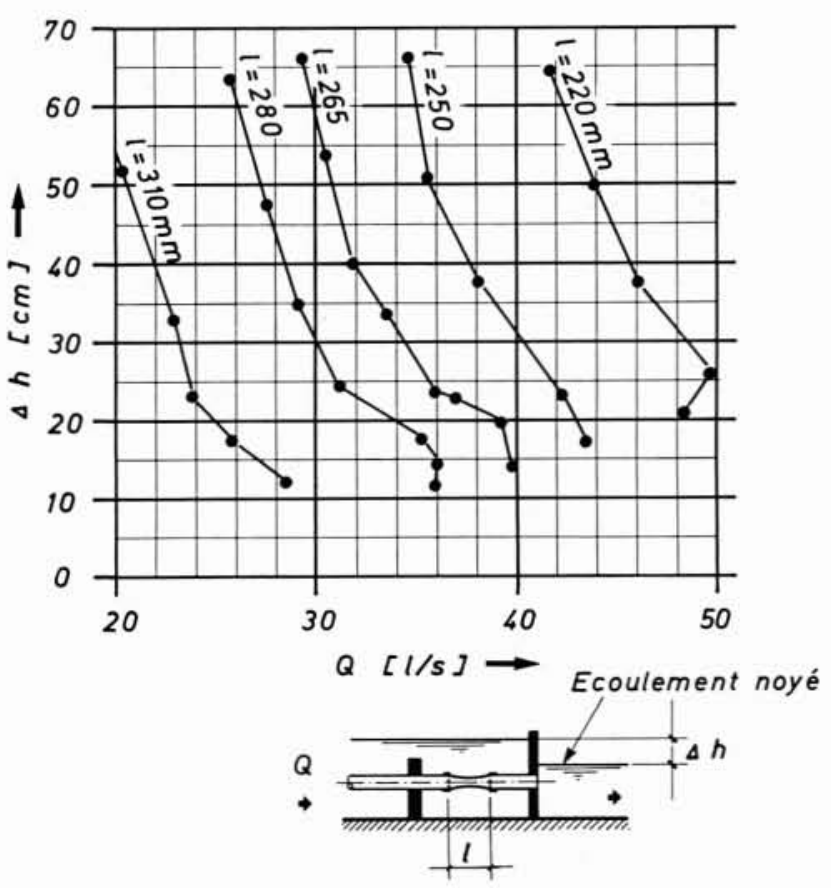

Figure 5. - Type IV: courbes des débits mesurés, avec écoulement aval noyé.
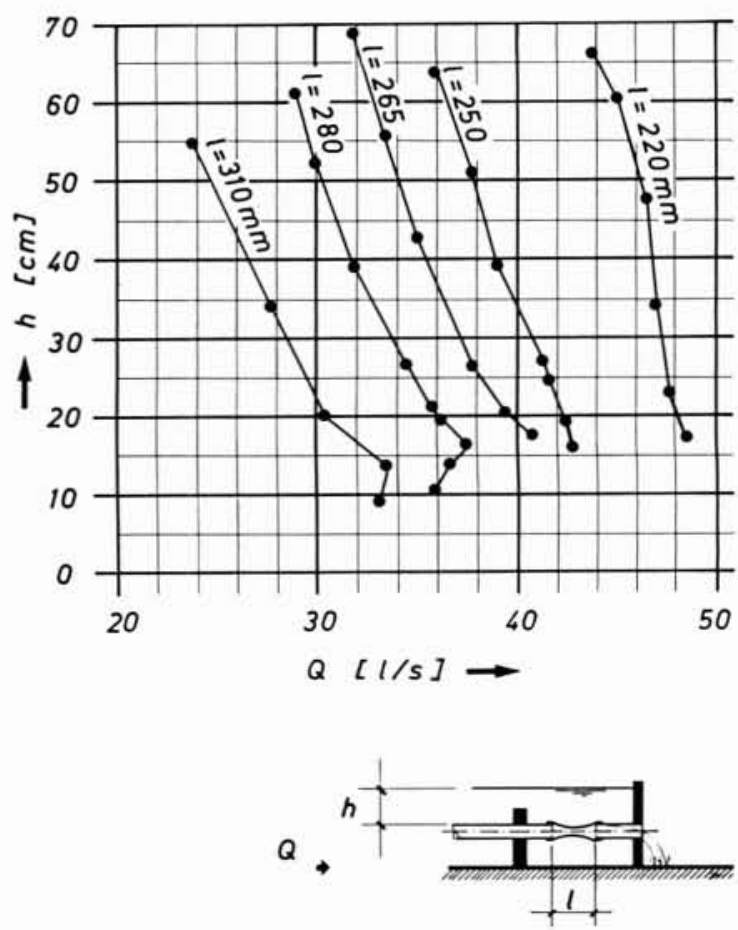

Figure 6. - Type IV: courbes des débits mesurés, avec écoulement aval dénoyé.

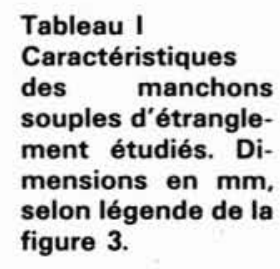

Tableau I

souples d'étrangle-

ment étudiés. Di-

selon légende de la

figure 3.

\begin{tabular}{|c|c|c|c|c|c|c|}
\hline Type & I & II & III & IV & v & IV \\
\hline \multicolumn{7}{|l|}{ Tuyau } \\
\hline $\mathrm{D}_{1}$ & 100 & 125 & 150 & 200 & 250 & 300 \\
\hline $\mathrm{d}_{1}$ & 10.25 & 11.25 & 12.25 & 14.5 & 16.75 & 18.75 \\
\hline $1_{1}$ & 340 & 340 & 340 & 340 & 340 & 340 \\
\hline $1_{2}$ & 850 & 830 & 800 & 980 & 920 & 800 \\
\hline \multicolumn{7}{|c|}{ Manchon souple } \\
\hline D & 120.5 & 147.5 & 174.5 & 229 & 283.5 & 337.5 \\
\hline d & 1 & 2 & 3 & 4 & 5 & 6 \\
\hline 1 (de à) & $100 / 180$ & $130 / 190$ & $170 / 230$ & $220 / 310$ & $250 / 340$ & $300 / 390$ \\
\hline L & 460 & 500 & 540 & 620 & 700 & 780 \\
\hline \multicolumn{7}{|c|}{ Ouvrage d'étranglement } \\
\hline Ltot & $1290 / 1370$ & $1300 / 1360$ & $1310 / 1370$ & $1540 / 1630$ & $1510 / 1600$ & $1440 / 1530$ \\
\hline
\end{tabular}

Au total, six types de manchon souple d'étranglement de 4 à 5 longueurs différentes ont été examinés. Sur la base d'essais préliminaires, plusieurs dimensions ont pu être fixées a priori. Le tableau I en donne une vue d'ensemble. Les mesures effectuées ont porté sur les courbes de débit. Ceci signifie que pour chaque manchon, le débit, le niveau amont et le niveau aval ont été relevés. D'autres particularités - comme les vibrations, la formation de tourbillons, l'effet de la distance du radier et des bords du canal (voir sous 3) - ont été l'objet d'une appréciation essentiellement visuelle. Les résultats d'essais ont pu être remis à
Universal Ingénieurs-Conseils SA sous la forme d'un rapport en juillet 1980 (voir VAW 1980).

Comme exemple d'analyse, la figure 5 montre les courbes de débit d'un manchon du type IV pour des longueurs de 220, 250, 265, 280 et $310 \mathrm{~mm}$. Il convient de remarquer que les erreurs de mesure peuvent atteindre $3 \%$ de la valeur portée en abscisse. A titre de comparaison, la figure 6 montre les résultats des mesures pour un écoulement dénoyé à l'aval. Ceux-ci montrent une similitude étonnamment prononcée. 


\section{Problèmes particuliers}

\subsection{Le voilement du manchon}

Le voilement du manchon intervient aussitôt que la surpression dépasse une certaine valeur, dite pression critique de voilement. Si le manchon est relativement rigide ou respectivement la surpression modérée, comme c'est le cas pour ces essais, la forme du voilement est très régulière. Dans d'autres cas, on a pu observer des déformations fortement irrégulières, asymétriques par rapport à l'axe du manchon.

La forme du voilement n'a pas été mesurée au cours des essais mais seulement observée visuellement. Comme le montre la figure 7, le nombre des bosselures de voilement sur le pourtour du manchon varie entre 3 et 6 . Ce nombre coïncide presque sans exception avec le nombre que l'on peut déterminer sur la base de la théorie de la déformation des coques élastiques (voir Vischer 1979); il ne dépend pratiquement que des dimensions du manchon.

Ainsi, l'effet d'étranglement du manchon est fonction simultanément de la rigidité de ce dernier et de la forme du voilement. Le diagramme de la figure 7 met en évidence ce phénomène : pour une qualité et une épaisseur de caoutchouc données, la courbe de débit et le nombre de bosselures varient avec la longueur libre du manchon.
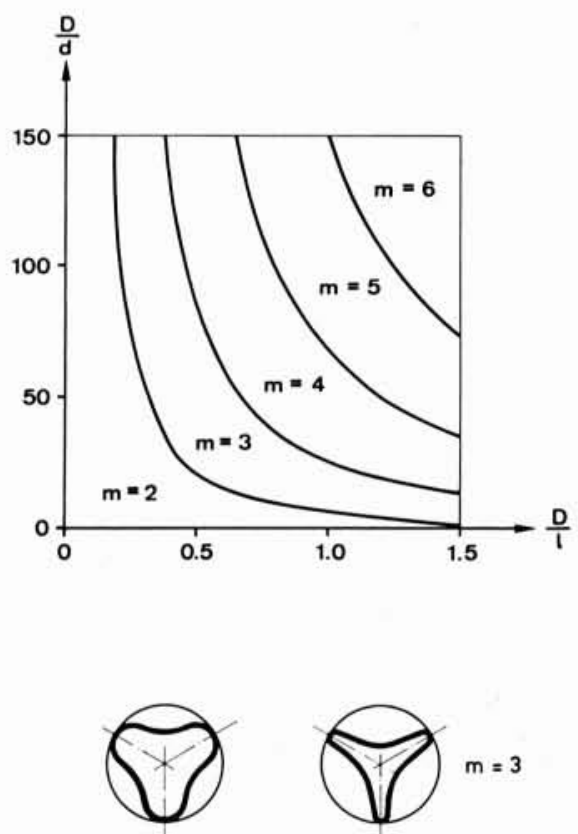

$m=3$
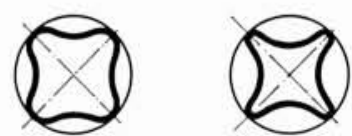

$m=4$
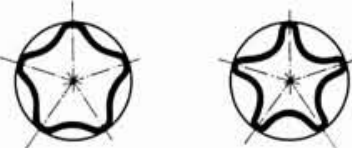

$m=5$

Figure 7. - Variations du nombre des bosselures dues au voilement en fonction des dimensions du manchon (à gauche). Formes des bosselures du manchon (à droite).

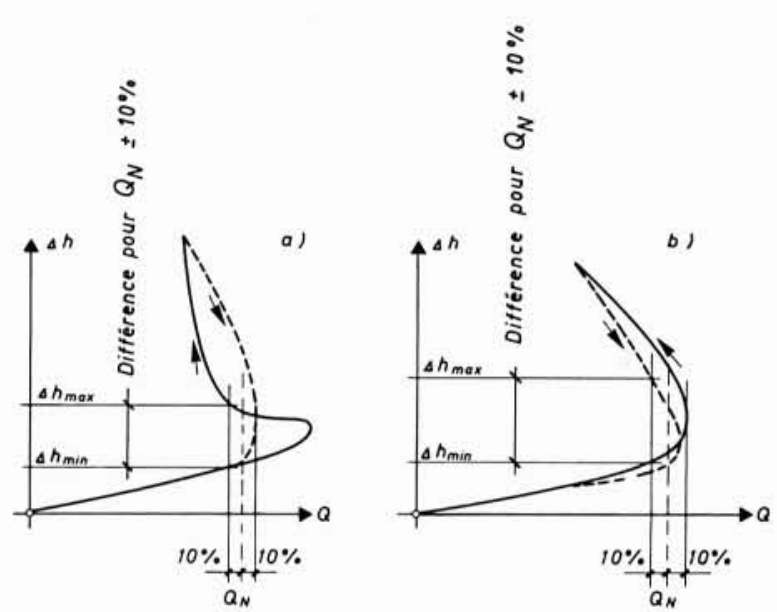

Figure 8. - Courbes typiques des débits lorsque la différence de niveau amont-aval est croissante puis décroissante : effet d'hystérésie. Définition du débit nominal $\mathrm{Q}_{\mathrm{N}}$.

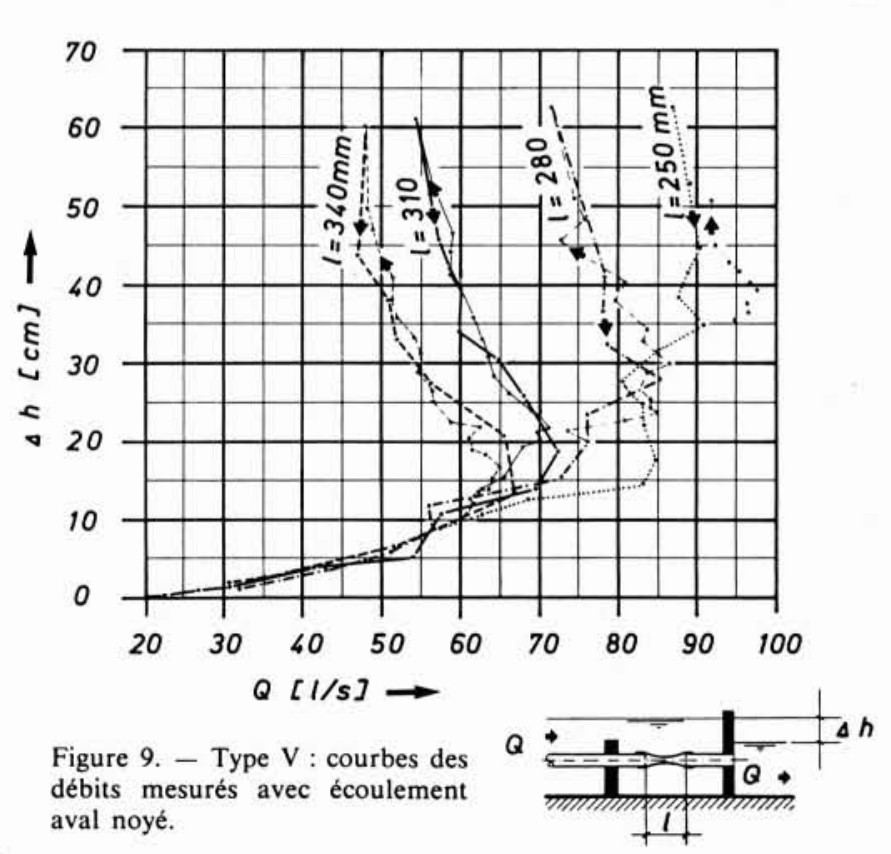




\subsection{Effets d'hystérésie}

Les figures 5 et 6 montrent des courbes de débit obtenues avec des surpressions croissantes, c'est-à-dire avec un niveau amont de plus en plus élevé. Ces courbes ne correspondent pas rigoureusement à celles obtenues lors d'un abaissement consécutif du niveau amont. En général, des différences apparaissent. Elles sont montrées schématiquement dans la figure 8 et illustrées par l'exemple du manchon du type $\mathrm{V}$ dans la figure 9.

Cet effet d'hystérésie est essentiellement lié aux propriétés du caoutchouc qui présente, sous traction, une hystérésie évidente dans son diagramme contrainte-déformation. D'autre part, il reflète également l'apparition d'un phénomène hydraulique lié à la position du point où l'écoulement décolle de la paroi.

Il convient de remarquer qu'à l'effet d'hystérésie des courbes de débit de la figure 9 viennent se superposer les erreurs de mesure, erreurs pouvant atteindre, comme indiqué plus haut, jusqu’à $\pm 3 \%$ de la valeur des débits.

\subsection{Fluage}

Des essais de longue durée ont permis d'observer des déformations marquées dues au fluage. Ainsi, un manchon souple d'étranglement du type IV, soumis pendant 30 heures à la sollicitation maximale, a subit une déformation due au fluage ayant entraîné une diminution de 4 à $6 \%$ du débit transité. Cette déformation a cependant disparu après que l'état de sollicitation ait été interrompu. Une semaine plus tard, le manchon sollicité à nouveau a permis d'obtenir une courbe de débit pratiquement semblable à la courbe initiale. Les déformations plastiques sont restées très petites. Elles ne proviennent pas uniquement de la plasticité du caoutchouc mais, dans une certaine mesure, également de la relaxation des fixations du manchon.

\subsection{Vibrations et pulsations}

Des vibrations et des pulsations ont pu être parfois observées pendant les essais.

Les vibrations se manifestent par un tremblement ou un battement du manchon présentant de faibles amplitudes et des fréquences élevées. Le débit n'est pratiquement pas influencé par ce phénomène peu prononcé. En l'absence d'une arête bien marquée, le point de décollement de l'écoulement n'est pas rigoureusement déterminé et effectue un mouvement pendulaire qui est à l'origine de ces vibrations (voir figure 2).

De telles vibrations n'ont été constatées qu'avec les manchons de petites dimensions. Il n'a guère été possible d'en empêcher l'apparition. Les conséquences pour le manchon restent cependant sans gravité car le caoutchouc peut sans autre résister à ces variations de charge. A long terme, un relachement des brides n'est pas à exclure.

Les pulsations provoquent une fermeture et une ouverture du manchon à un rythme de basse fréquence qui engendre une discontinuité du débit. Ce comportement est dû à un engorgement soudain de la sortie du manchon. Il s'ensuit un coup de bélier qui augmente l'étranglement jusqu'à l'interruption presque totale du débit. La disparition du jet entraîne alors l'ouverture du manchon et le passage consécutif du débit en provoque à nouveau la fermeture brutale.
Au cours des essais, de telles pulsations n'ont été observées qu'en présence de longs manchons. Le phénomène est beaucoup plus fréquent pour des orifices noyés que pour des orifices dénoyés. Les pulsations doivent être considérées comme préjudiciables au système car

- elles provoquent un débit discontinu:

- elles sollicitent fortement le caoutchouc;

- le manchon peut se libérer des brides de fixation.

L'utilisation de longs manchons est par conséquent à éviter (voir § 4.2).

\subsection{Formation de tourbillons}

Avec presque tous les types et toutes les longueurs de manchon examinés, les essais ont montré l'apparition d'un tourbillon localisé à l'entrée de l'ouvrage. Ce tourbillon apparaît pour un niveau amont moyen et est accompagné le plus souvent d'un entraînement d'air. Il n'affecte ni le débit, ni l'étranglement. Son influence sur les vibrations et sur les pulsations n'a pu être toutefois complètement éclaircie.

\subsection{Constitution de l'entrée}

Dans le cadre des essais entrepris, les conditions d'écoulement à l'entrée ont été soumises à un examen détaillé. Il s'est ainsi avéré que la distance de l'entrée aux parois, respectivement de l'entrée au radier n'a aucune influence sur le fonctionnement du manchon proprement dit. Par contre, l'intensité du tourbillon d'entrée dépend de la configuration choisie (voir $\S 3.5$ ).

La section d'entrée a été modifiée notamment par l'adjonction d'une trompette de forme hydrodynamique. L'amélioration obtenue reste minime, le débit n'ayant pu être augmenté ainsi que de 3 à $4 \%$.

De ce fait, il paraît recommandé de réaliser une entrée aussi simple que possible, conçue par exemple selon le modèle de la figure 2 . Les ouvrages de répartition étant très nombreux dans les installations d'irrigation, ceux-ci doivent le plus possible être construits de façon simple et économique.

\subsection{La forme de l'étranglement}

Des modifications apportées à la configuration du manchon et, par conséquent, à la forme de l'étranglement ont également été étudiées. Des nervures de raidissement diverses, longitudinales ou transversales ont été collées sur le manchon. Dans d'autres cas, des structures rigides ont été placées à l'intérieur de celui-ci. On a cherché en ce faisant à poursuivre deux objectifs :

- la diminution ou la suppression des instabilités, c.à.d. des vibrations et des pulsations;

- l'amélioration des courbes de débit dans le sens d'une transformation de la pente négative en une pente positive.

Ces objectifs ont été atteints. Toutefois, l'amélioration possible des caractéristiques du manchon entraîne une complication excessive du dispositif et reste ainsi peu attractive.

Pour les applications dans les canaux d'irrigation prévues ici, la forme la plus simple du manchon, soit la forme cylindrique, peut être recommandée. 


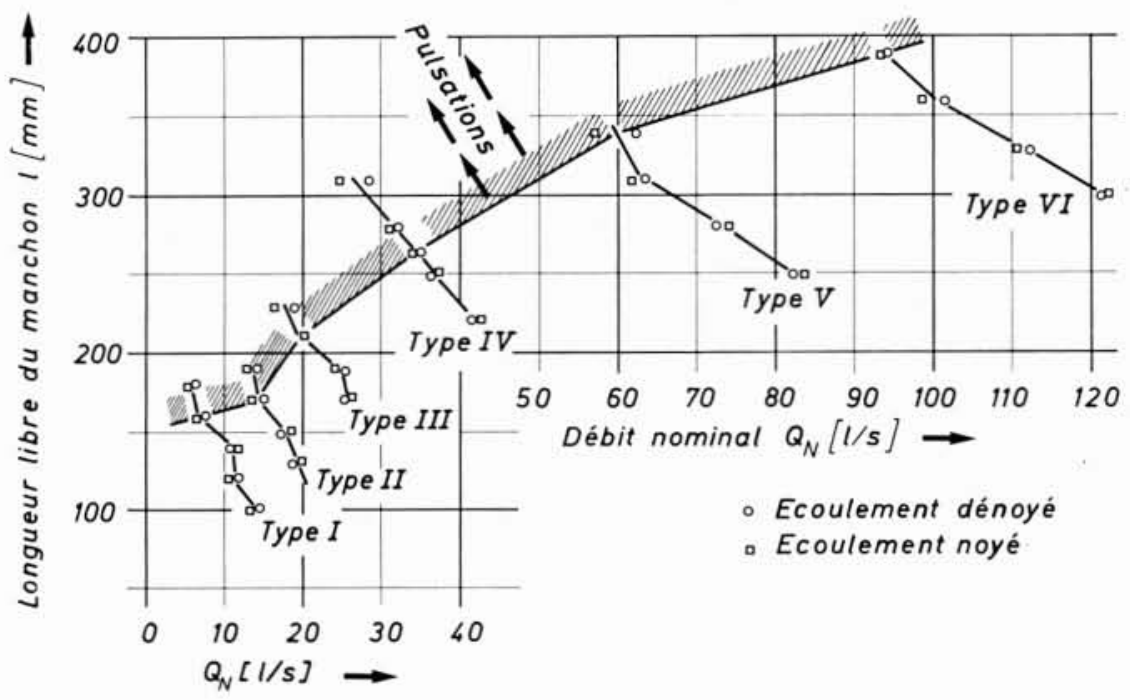

Figure 10. - Diagramme de dimensionnement du manchon souple d'étranglement. Le type et la longueur libre du manchon sont donnés en fonction du débit nominal $Q_{N}$.

\section{Dimensionnement}

\subsection{Définition du débit nominal}

La méthode suivante a été appliquée pour définir le débit nominal et le domaine des variations de pression propres à chaque manchon :

La courbe de débit a d'abord été réduite de $6 \%$ en abscisse pour compenser les effets du fluage du manchon (voir § 3.3). Le débit maximal $Q_{\max }$ a été ensuite relevé et défini comme $110 \%$ de la valeur du débit nominal $Q_{N}$. Le débit $Q_{N}$ est donc la valeur de $Q_{\max }$ réduite de $1 / 11^{\text {c}}$. Le débit minimal est défini alors comme représentant $90 \%$ de la valeur de $Q_{N}$. Il délimite, selon la figure 8 , un domaine de variation de pression dans lequel le manchon d'étranglement peut être mis en fonction sans que son débit nominal subisse des écarts supérieurs à $\pm 10 \%$.

Pour une courbe de débit comme celle de la figure $8 \mathrm{~b}$, cette méthode ne présente aucune difficulté. Par contre, pour la courbe de débit de la figure $8 \mathrm{a}$, elle requiert une certaine adaptation; la pointe excessive mesurée pour des niveaux amont croissants doit être négligée car elle n'apparaît pas en cas de sollicitation prolongée du manchon.

La méthode présentée ci-dessus est encore susceptible de développements et de perfectionnements. Il ne s'agit donc ici que d'une première approche du problème.

\subsection{Le diagramme de dimensionnement}

Le diagramme de la figure 10 présente tous les débits nominaux déterminés par l'essai en fonction de la longueur libre du manchon. Phénomène intéressant, les points correspondant à un écoulement dénoyé à l'aval sont situés sur la même courbe que ceux correspondant à un écoulement noyé. Sur ce même diagramme est également reportée la courbe limite au-delà de laquelle apparaissent des pulsations de l'écoulement.

Ce diagramme peut être considéré comme un diagramme de dimensionnement. Pour un débit nominal fixé (débit de dimensionnement), il indique le type et la longueur du manchon à adopter. Les courbes données ne couvrent toutefois pas la totalité des débits possibles car des diamètres intermédiaires des tuyaux en fibrociment n'ont pas pu être obtenus. Ces lacunes pourraient être comblées par l'utilisation de membranes en caoutchouc d'épaisseur $1.5,2.5,3.5,4.5$ ou $5.5 \mathrm{~mm}$. De telles membranes ne sont pas encore commercialisées actuellement.

L'alternative consiste alors à faire fonctionner en parallèle deux ou plusieurs manchons choisis parmi les différents types étudiés. 


\subsection{Exemples de dimensionnement}

Exemple 1: Pour un débit nominal de $20 \mathrm{~V}$ s, quel type de manchon convient-il de choisir et quelle doit être sa longueur libre?

Du diagramme de la figure 10 , on obtient une longueur de $122 \mathrm{~mm}$ pour un manchon du type II et une longueur de $209 \mathrm{~mm}$ pour un manchon du type III. Avec une tolérance de débit $Q_{N} \pm 10 \%$, la différence de niveau entre l'amont et l'aval peut être tirée des courbes de débits déjà réduites de $6 \%$, données pour les manchons de types II et III. (Ces courbes ne sont pas montrées à nouveau ici, voir VAW 1980). La différence de niveau minimale est de $21 \mathrm{~cm}$ pour le type II et de $12 \mathrm{~cm}$ pour le type III.

Cet exemple montre un cas peu fréquent où le diagramme de dimensionnement donne deux solutions possibles. Si l'on veut à tout prix éviter les pulsations, on choisira le manchon le plus court, soit du type II. Si le système doit fonctionner avec une petite différence de niveau, le choix se portera sur le manchon du type III.

Exemple 2: Quel manchon choisir pour un ouvrage de réglage de $50 \mathrm{l} / \mathrm{s}$ ?

Le diagramme de dimensionnement présente une lacune pour le débit de $50 \mathrm{l} / \mathrm{s}$. Si l'on veut s'en tenir à la série des manchons essayés, on peut utiliser deux manchons dont le débit nominal est de $25 \mathrm{l} / \mathrm{s}$. Il s'agit ainsi de 2 manchons du type III présentant une longueur libre de $188 \mathrm{~mm}$. Cet exemple montre que le domaine d'application des 6 types de manchons étudiés est assez étendu. Mais il convient de rappeler que dans le cadre de ces essais, la différence de niveau entre l'amont et l'aval a été limitée à $0.70 \mathrm{~m}$, ce qui correspond cependant aux situations rencontrées normalement dans les systèmes d'irrigation.

\section{Utilisation du manchon souple d'étranglement dans des réseaux d'irrigation}

\subsection{Généralités}

Par les caractéristiques hydrauliques décrites ci-dessus, le manchon souple d'étranglement peut être classé comme module-type d'appareil assurant la répartition des débits constants indépendamment des fluctuations du niveau d'eau amont et aval. Il pourrait donc être installé dans des prises d'eau, en sortie de bassin de retenue ou à l'entrée des canaux d'irrigation de répartition, soit par exemple entre le réseau secondaire et le réseau tertiaire ou à l'entrée du canal d'irrigation proprement dit.

\subsection{Choix du type du manchon souple d'étranglement et conception de l'ouvrage}

Le dimensionnement est présenté au chapitre précédent. Par la combinaison de deux ou même de plusieurs types de manchon, on peut non seulement combler les lacunes subsistant pour les débits qui n'ont pas encore été contrôlés par l'essai, mais on dispose également d'un système de réglage des quantités d'eau variant avec les différentes périodes d'irrigation.

La figure 11 donne un schéma vu en perspective d'une implantation-type de deux manchons souples d'étranglement en tête d'un canal d'irrigation. L'ouvrage peut être situé dans une des berges du canal d'adduction. Les manchons sont incorporés dans un puisard, qui est ouvert vers le canal d'adduction pour assurer la sollicitation des manchons. La paroi réduite du puisard côté aval est munie de vannes. Tous les travaux du génie civil peuvent être effectués soit en béton normal soit en maçonnerie.

Le revêtement des canaux à l'entrée et dans le bassin de dissipation d'énergie peut être réalisé en pierre. Sous des conditions normales où l'on veut assurer des pertes de charges minimales, on prévoit l'emplacement de l'ouvrage tel qu'un écoulement noyé s'installe à l'aval. Comme indiqué plus haut, le système fonctionne également avec un écoulement libre à l'aval, par exemple en combinaison avec le bassin d'amortissement d'un ouvrage de chute. En cas de nécessité, l'ouvrage peut naturellement être combiné avec un ouvrage de régulation.

Il est conseillé de couvrir le puisard avec des planches afin de protéger les manchons contre une altération éventuelle.

\subsection{Essais in situ}

De 1980 à 1982, Universal Ingénieurs Conseils SA a testé le manchon souple d'étranglement sous des conditions tropicales, dans un ancien système d'irrigation du périmètre Eyadema à Mango, Togo.

Les manchons, d'un débit $Q_{N}=40 \mathrm{l} / \mathrm{s}$, ont été incorporés dans des structures de répartition existantes. La figure 12 montre qu'il est sans autre possible d'utiliser un dispositif très simple.

Les résultats acquis sont très encourageants et montrent que :

- la mise en place peut être effectuée par la main d'œuvre locale. Le coût de l'ouvrage est très bas;

- le bon fonctionnement des manchons en caoutchouc n'est pas influencé par le climat tropical. Le caoutchouc n'a pas été attaqué par des prédateurs, mais devrait être protégé contre la curiosité intempestive des villageois; - la vibration des tuyaux empêche la formation de dépôts solides. 


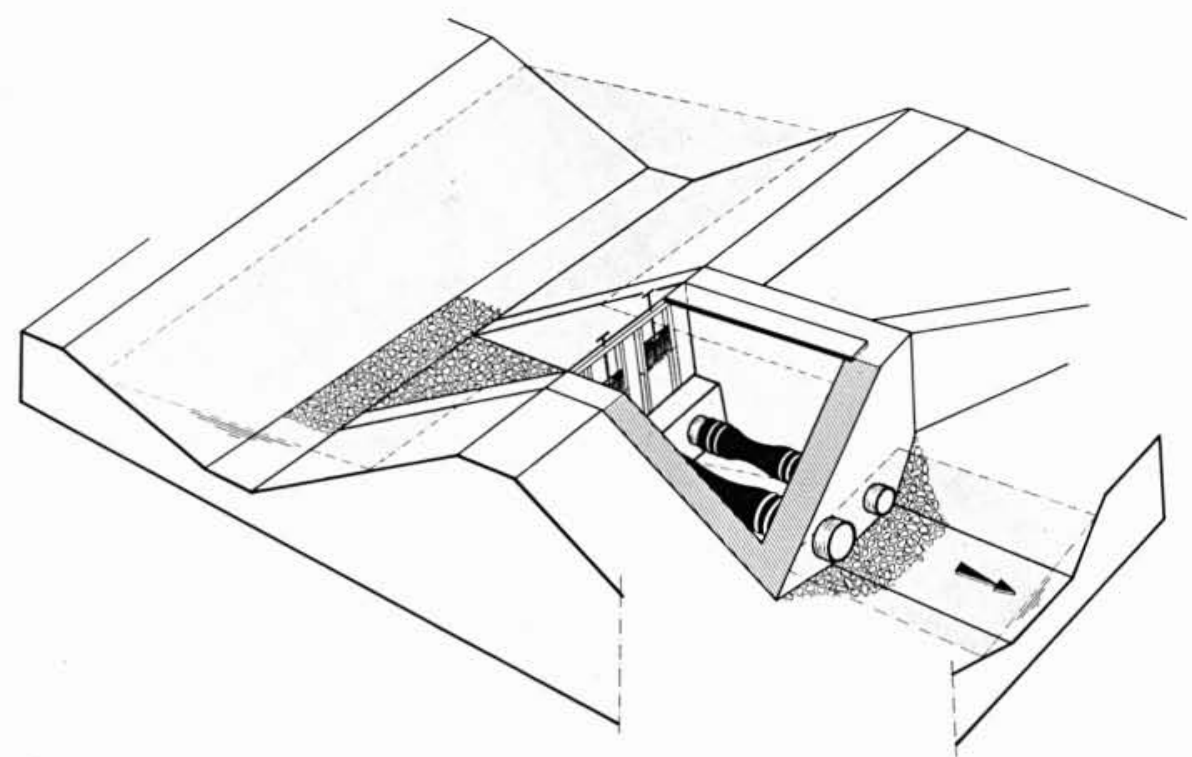

Figure 11. - Perspective de l'implantation-type de deux manchons souples d'étranglement.

Figure 12. - Implantation du type IV dans un petit périmètre d'irrigation en République Togolaise.

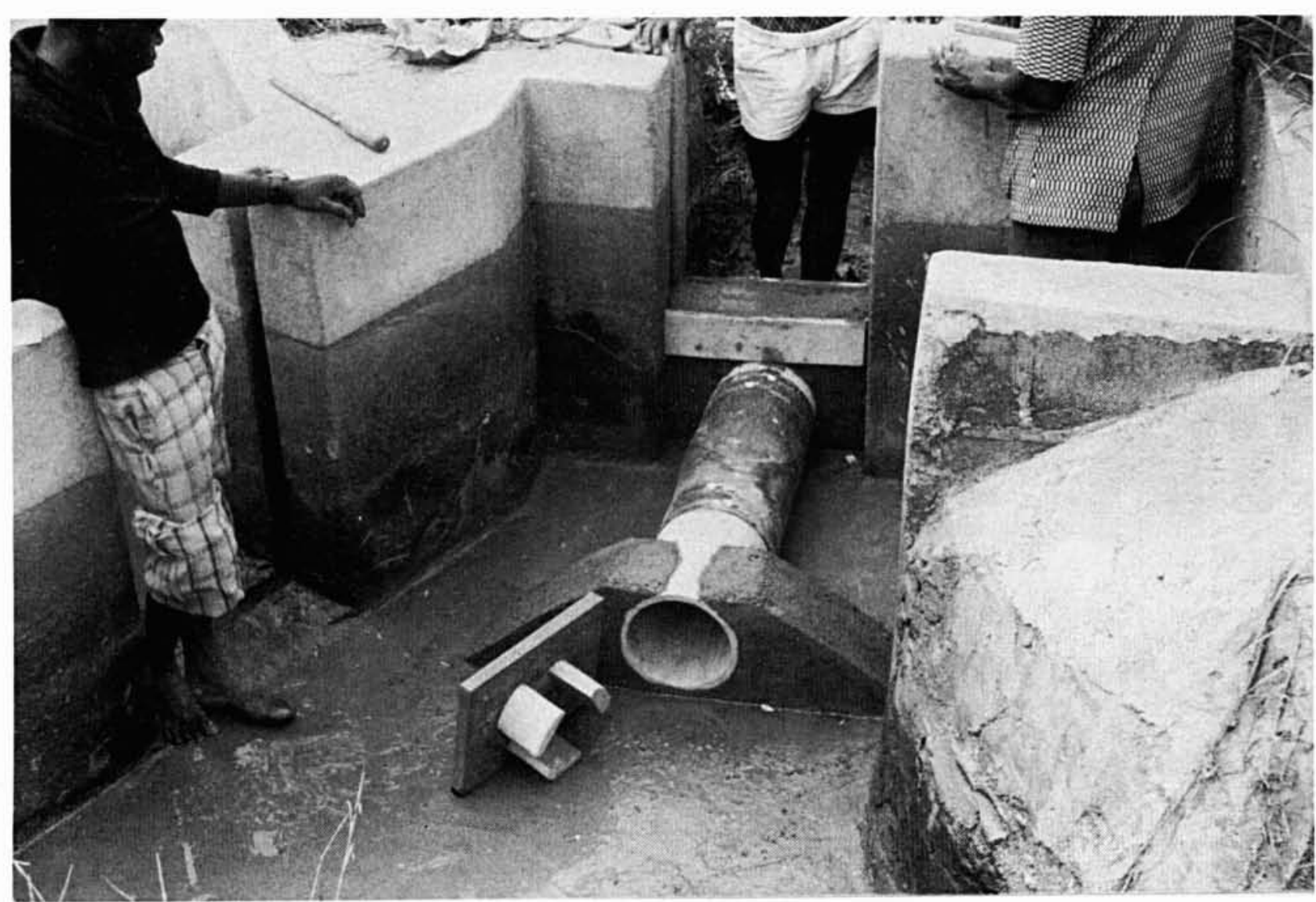




\section{Résumé}

Avec le manchon souple d'étranglement, on dispose d'un nouvel organe autorégulateur de répartition des débits constants dans les réseaux d'irrigation. Sous certaines conditions, il peut présenter une alternative valable aux systèmes de répartition existants. Sa configuration est simple, ce qui se traduit par des coûts modestes de réalisation.

Le présent article explique le principe de fonctionnement du manchon souple d'étranglement et décrit les essais de laboratoire menés avec une série de prototypes. Il traite également certains problèmes spécifiques, tels que les effets d'hystérésie et de fluage inhérents au tuyau de caoutchouc utilisé, ainsi que l'apparition de vibrations et de pulsations. Enfin, il propose un procédé de dimensionnement basé essentiellement sur un diagramme simple et facilement compréhensible.

Il faut se garder de croire cependant que tous les problèmes posés par l'application du manchon souple d'étranglement sont déjà résolus. Comme dans tout nouveau développement, il va de soi que plusieurs questions restent encore en suspend. Cependant, les expériences faites après plus de 2 ans d'utilisation du système dans des conditions tropicales sont encourageantes. Néanmoins, la réalisation d'essais supplémentaires en laboratoire et in situ s'avère nécessaire. Les réactions et les suggestions des lecteurs à ce sujet seraient fortement appréciées.

\section{Bibliographie}

[1] VisCHER D. (1979). - Die selbsttätige Schlauchdrossel zur Gewährleistung konstanter Beckenausflüsse. (Utilisation du manchon d'étranglement autorégulateur pour le maintien de débits d'écoulement constants à la sortie d'un réservoir), (en allemand) Revue "Wasserwirtschaft ", $69 \mathrm{Jg}$., Heft 12/1979. Frankhe Stuttgart.

[2] VAw, Laboratoire de Recherches Hydrauliques, Hydrologiques et Glaciologiques de I'EPF Zurich (1980)
Rapport $n^{\circ} 766$ à l'intention de la Société Universal SA Ingénieurs Conseils, Bâle, juillet 1980 (en allemand, non publié).

[3] UnIVERSAL INGÉNIEURS Conseits (1982): The automatic throttle hose, an irrigation outlet with near constant discharge. (Le manchon d'étranglement autorégulateur, un organe d'irrigation à débit quasi constant), (en anglais). Leaflet 1982, Universal, POB 4010 Bâle, Suisse.

Adresses des auteurs :

- Daniel L. VISCHER, Professeur de constructions hydrauliques,

Laboratoire de Recherches

Hydrauliques, Hydrologiques

et Glaciologiques (VAW)

Ecole Polytechnique Fédérale de Zurich (EPF)

$\mathrm{CH}-8092$ Zurich (Suisse)

- Paul J. PEter, Ingénieur en génie rural.

Universal Ingénieurs Conseils SA.

Malzgasse 30

CH-4010 Bâle (Suisse) 